\title{
Supporting Group and Personal Memory in an Interactive Space for Collaborative Work
}

\author{
Mari Tyllinen and Marko Nieminen \\ Aalto University School of Science, P.O. Box 15400, FI-00076 AALTO, Finland \\ \{mari.tyllinen, marko.nieminen\} aaalto.fi
}

\begin{abstract}
This paper reports the findings from our research on constructing the memory functions of DiWa (Digital War room), an interactive collaboration environment to support work especially with digital information. The aim is to identify the current organizational and personal practices of storing and retrieving meeting information. We report findings from a field study conducted in selected units of four organizations and results from the initial stage of a survey on the users of our collaboration environment. Our findings include that organizational practices and systems for storing meeting information are varied and they are not used systematically; personal practices still largely involve pen and paper notes; and the need to return to meeting information exists.
\end{abstract}

Keywords: Collaborative work, Interactive collaboration environment, User study, Meeting capture.

\section{Introduction}

Working in teams is business as usual in most organizations and companies regardless of the field of work. The level of collaboration in teams may vary depending on the work culture or objective. In our work, special consideration is put into studying the capabilities of the interactive space to support the capturing and recalling of events and issues that have been addressed during the joint meetings.

Researchers have been interested in team work from many perspectives during the last decades. Working in teams in office-type surroundings has also been the subject of studies e.g. in computer-supported collaborative work. From the technological perspective several projects over the years have involved constructing technologically enhanced meeting rooms or interactive spaces to support working in teams (e.g. [1-3]). Researchers have also focused their efforts on building systems to support meeting capture and later browsing and retrieval of the captured data (e.g. [4-5]). However, these systems and facilities have still not become part of everyday activities in the industry. It has been suggested that this is in part due to a lack of a user-centred approach in the development [6].

In this paper we describe research activities involving the user-centred development of an interactive collaboration environment. Our DiWa project involves four public and private organizations from industrial manufacturing as well as construction 
and zoning. The project started with a literature review, continued with a field study and requirements elicitation after which the first prototype of the environment was constructed.

This prototype implemented the infrastructure that provided the initial facilities to support collaborative work. Instead of being considered as the final solution, the technical realization of the interactive space has provided us a modifiable research instrument that can be used to study various features in an iterative and interactive way. The initial features of our enhanced co-located collaboration environment originated from the literature review and the initial findings or the field study. The first components of the interactive space were large interactive touch-screens with remote presentation capabilities, an environment-specific wireless networking infrastructure with shared file storage. The prototype also implemented the basic functionality of logging user actions such as saving information on opened documents. (See Figure 1)

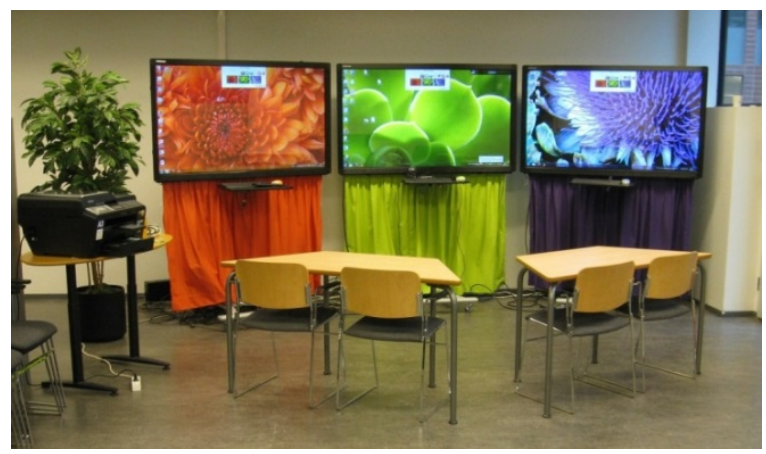

Fig. 1. Our co-located collaboration environment

During fall 2012 our interactive environment and software prototype was used in the university premises. During this time our interactive space was used by students and researchers for different purposes such as software development team work, research workshops, presentations, and teaching.

In this paper, we focus on our research activities that aim at defining the meeting capture, browsing and retrieval functionality which are intended to help the collaborating group in their recurring work. More specifically, we aim at gaining increased understanding on how to supplement and expand personal and organizational practices for storing and retrieving information from meetings. We aim to do this by identifying those aspects of collaborative work that are important for recalling recurring group activity and by finding ways of technologically enhancing current memory aiding practices such as note-taking. The specific research question of our study is as follows:

What are the current personal and organizational practices for storing and retrieving meeting information?

The answers to this question help us in defining user-originated memory support features for the interactive collaboration environment. We will also conclude and 
discuss such prerequisites that would enable the introduction and broader utilization of memory support functions in collaborative environments.

\section{$2 \quad$ Related Research}

Technologically enhanced meeting rooms that support working in teams have been studied by several researchers. The iRoom [3] is a meeting room enhanced with large touch displays both on walls and on a table. The room includes support for multiple personal computing and communication devices that integrate with each other. The IMPROMPTU interaction framework [7] supports collaborative work in a multidisplay environment. The framework enables sharing of information across multiple displays concurrently. WeSpace [2] is an interactive space combining a large display wall and a multi-user multi-touch table for use by scientists. The space is especially designed for easy access with personal devices and applications to begin a shared situation where visual data from multiple participants is examined collaboratively. The NiCE Discussion Room [1] takes a slightly different approach in focusing on integrating digital and paper tools into a meeting room design.

Our focus is in supporting the memory of meeting participants with specific functionality within our interactive collaboration environment. A number of previous works have implemented memory supporting functionality for capturing and retrieving meeting data. Whittaker et al. [6] have presented a categorization of retrieval systems or meeting browsers. They present four categories: audio focused, video focused, artifact focused and discourse focused. TeamSpace [4] is a team interaction space that among other things supports capture and access of virtual meetings. The capturing and access is time-based. Jaimes et al. [5] have constructed a meeting retrieval system based on a visuospatial user interface and dynamic queries into the data. The MemTable [8] is a system based on a custom-built interactive tabletop. The retrieval is based on both searching and temporal browsing. A special feature is the ability to include information from the retrieval into a current meeting.

Whittaker et al. [6] have done research on the current practices of creating meeting records. Jaimes et al. [5] conducted a survey study on the reviewing of past meeting data especially regarding video capture. Plaue et al [9] researched the in-meeting sharing of information and found that the sharing practices with own laptops varied and appropriation of new technology depends on whether it adds value to existing practices. Our study focuses on current organizational and personal practices for capturing and retrieving meeting data.

\section{$3 \quad$ Methods and Data}

\subsection{Field Study}

The results in this paper represent a subset of a larger field study that contained the following themes: different everyday collaboration situations, their progress and significance, collaboration facilities and their use, media and artifacts related to 
collaboration as well as problems with collaboration. The field study consisted of observations, interviews and group interviews. The participants of our field study were employees from the organizations involved in our DiWa project including product development engineers, industrial designers, project managers, planning engineers and architects.

Our data for this paper originates from seven collaboration situations in which the participants were either completely internal (4) or involved external participants (3). The number of participants in these situations ranged from five to eight. The observations from the situations were written notes on the following viewpoints: (a) participants; (b) technology and its use; (c) layout of the space; (d) participants' movement within the space; (e) interaction between participants; (f) handled media and artifacts; (g) role of meeting in the decision making process; (h) references to shared past situations of information. Especially viewpoints (b) and (f)-(h) are relevant regarding the research question of this paper.

Additionally, our data consists of four individual interviews and four group interviews that had 18 participants. These interviews were conducted with employees in different roles in the studied units. Some of the participants were present in the earlier observed meetings. The interviews were conducted by two researchers; one researcher was responsible for transcribing and making detailed notes in real time while the other moderated the interview. The interviews were also audio recorded.

The themes of the semi-structured interviews were: (a) role and background of participants; (b) work tasks; (c) everyday collaboration situations; (d) facilities for collaboration; (e) media and artifacts used in the collaboration; (f) informal collaboration; (g) problems with collaboration.

The aims of our analysis that are reported in the results section of this paper were to identify: (a) current organizational practices of saving records; (b) the types of records saved; (c) the systems used for preserving and sharing records; (d) reasons and practices of creating personal records; (e) reasons and occasions for returning to past records. We classified 80 findings for (a), 60 findings for (b), 31 findings for (c), 14 findings for (d) and 19 findings for (e) from the field study data.

\subsection{User Survey}

In addition to the field study we conducted a user survey on personal memory support and managements practices with the users of our interactive space in the university premises. This survey is the initial stage of a survey study that will be continued during the piloting of our interactive space in the organizations involved in the DiWa project.

The survey is divided into before-meeting and after-meeting sections. It includes questions on the reasons, tools and forms of personal note-taking as well as reasons and strategies for returning to past meetings both at general and meeting-specific levels. The questionnaire consisted of open-ended questions (5) as well as questions about the frequency of different note-taking practices (4) including the returning to previous notes and memos. The questions of our survey are in part based on the study 
reported by Jaimes et al. [5] which focused on the use of different tools and strategies to review past meetings.

The questions in the before-meeting questionnaire were: 1) Do you take personal notes in meetings? 2) How often do you use these tools to make personal notes? 3 ) How often do you use the following ways to make notes? 4) Do you return to past meetings (i.e. to recall the event details, decisions, ...)? 5) How often do you use the following tools or strategies to return to past meetings?

The questions in the after-meeting questionnaire were: 1) How did you take the notes? 2) What information from your meeting would you imagine using/needing afterwards? 3) If you could access a complete record of everything that happened in meetings, would you be interested in returning to past meetings? Please evaluate the importance of the following reasons to return to past meetings.

Out of the 19 participants to the before-meeting-questionnaire, 7 represented the zoning organization and the rest were students and researchers of our university. Out of the 11 participants to the after-meeting-questionnaire, 3 represented the zoning organization and the rest were students and researchers.

\section{$4 \quad$ Results}

\subsection{Field Study}

Current Organizational Practices of Saving Records. Our field study reveals that in principle the studied organizations have ways and systems for saving records of meetings. However, these systems are not used systematically mostly due to a lack of commonly agreed upon practices which is reflected in the following answers:

"All project files are accessible where ever, there is no one specific way"

"There is a project folder, but there are no instructions for using it"

"You don't get caught for not documenting".

A significant amount of work is written or drawn on paper during meetings. These documents can be digitalized by scanning or photographing, but often these documents remain to be filed by individuals that collect them in personal binders.

The practice of writing minutes or a memo is not common, and even when it is done there are several problems involved in the process. The quality of the minutes is considered to be the responsibility of the secretary. There is always the risk of the secretary having a different understanding of the meeting than other participants. The minutes are usually written by hand and misunderstandings are only visible afterwards to the other participants. In two of the organizations consultants are usually responsible for writing the minutes. However, they are considered to be poor at producing them on time or at all. Good practices involved with minutes and memos were writing the memo together at the end of the meeting and saving it to the system as the meeting ended.

The practices involved in documenting decisions and action points also varied. Usually it was the responsibility of the persons in question to make note of the action 
points. Following comment illustrates this: "It varies whether they are written down or not. Even clear decisions are not always documented."

In general, meetings involve a lot of discussion and comments that are not saved in any way. The documenting of "right things" is considered difficult. However, our field study suggests that there is interest in being able to return to this information.

The Types of Records Saved. Based on our field study, the material that is handled in meetings can be in various digital file formats, digital files printed on paper, physical objects such as prototypes and drawings or writings on a flip chart or white board. The digital file formats are used so that the contents are not evident based on the format: examples of these are web links in a PDF or memo in a spreadsheet. The way to save public records varied: saving to some system or sending by email.

The Systems Used for Preserving and Sharing Records. Through our field study it was evident that most organizations have several different systems for saving and sharing records ranging from network drives to different types of systems for different types of records such as for project management and for ideation. Some of these systems have support for collaborative activities. Email was used commonly as a way to share and save information. These systems have several problems including: network drives easily become disorganized, documents in the systems are not up-to-date, management of user rights is difficult, finding relevant information afterwards is hard.

Reasons and Practices of Creating Personal Records. Based on our field study only few people take notes from collaborative situations consistently; most people take notes occasionally. Notes are taken especially when there are matters that concern the individual personally, most commonly such issues are action points. The notes taken by people are most often done by hand, either to personal notebooks or material that was distributed on paper during the meeting, such as agendas.

Reasons and Occasions for Returning to Past Records. In our field study people expressed the hope of being able to return to the documentation of past meetings. This documentation would also give structure to the next meeting, which was utilized in some organizations by following on the progress of previous decisions. However, most often than not, these documents were not available or not found easily. Based on our study it also seems that special pictures, conceptualizations or meaningful ideas that have been developed in earlier meetings and have raised special interest leave a memory trace without the details. This prompts the need to return to the documents.

\subsection{User Survey}

Taking Personal Notes in Meetings. Ten out of the 19 respondents answered that they do take notes, five take notes sometimes, three don't take notes and one did not answer. The reasoning that they offered for taking notes seem to fall in two categories: for remembering (7) and collecting personal action points or tasks (3). Two respondents answered that taking notes interferes with participating in the meeting. 
Some people also feel they need to take notes to get an overview of the meeting for learning in the moment or for checking afterwards.

In the after-meeting questionnaire, however, only four out of 11 respondents reported taking notes. The main reasoning for not taking notes was that the meeting involved hands-on work.

Used Tools and Ways for Making Notes. Out of the five options (computer, digital camera, pen and paper, phone and other) the most used tool was pen and paper: 12 respondents answered that they use it often or always. The least used tool was phone: 11 respondents answered that they use it rarely or never. One respondent identified the iThoughts HD application for iPad as something he uses always. Out of the six options (drawing, taking pictures, video recording, voice recording, writing and other) the most used way of taking notes was writing: 17 respondents use if often or always. The least used ways of taking notes were video and voice recording: 19 and 17 respondents respectively use them rarely or never. One respondent identified his way of making notes to be mind maps.

In the after-meeting questionnaire, however, from the four out of 11 respondents that took notes three reported having used a computer and only one taking notes by hand.

Returning to Past Meetings. Twelve out of the 19 respondents answered that they return to past meetings, four return sometimes, one does not return and two did not answer. The reasoning that they offered for returning fell into the following categories: remembering details or discussions (6), preparing for the next meeting (3), checking for personal action points (2) and sharing information to others (1). Two respondents also review their notes for reflecting their thoughts further.

Out of the nine options (asking someone, common notes, distributed documents, images of white board/flip chart, personal notes, photographs, video recordings, voice recordings and other) for returning to past meetings, the most used tool was personal notes: 14 respondents answered that they use it often or always. Distributed documents were also quite common for returning: 9 respondents used them often or always. The least used tools were video and voice recordings: 18 respondents answered they use them rarely or never.

In the after-meeting questionnaire ten respondents identified the following possible reasons and ways for returning: needing details and discussions (6), using the presented or produced documents (3) and checking personal action points (1).

Possible Reasons for Returning to Past Meetings. Finally, respondents were asked to imagine that they would have a complete record of everything that happened in the meeting in different media formats (text, voice, pictures...) and rate the importance of reasons to return to past meetings. They were given ten options: (a) have accurate record of meetings; (b) keep in mind what customers or key persons said; (c) verify the truth when memory and descriptions are inconsistent; (d) reexamine other person's speech and its context to correctly understand meaning or intention; (e) listen to a portion of speech not understood; (f) listen to a portion of speech not heard; (g) know 
the results of discussions of ideas from earlier meetings with respect to current interest or problem; (h) obtain proof when someone denies having said something; (i) recall an idea that I had during a meeting; (j) check the consistency between present and earlier presentations.

Out of the 10 respondents to this question, seven respondents rated options (b) and (i) as strong or very strong reasons to return. Eight respondents rated option (h) as the least likely reason for returning.

\section{$5 \quad$ Memory Support Functionality}

From Personal to Shared. Despite the finding that only few persons in meetings tend to take notes there are usually some that do. Without too much additional burden to the participants this provides a possibility to improve the documentation level with little effort through transforming the personal note-taking into a shared activity. In addition to sharing personal notes with other participants, the note-taking activity in itself may be transformed into group activity. In a group setting, shared note-taking may be considered as less burdening for an individual. Our collaboration environment is designed to support this in several ways: by making sharing files with others easy through a shared repository, by providing an easy two-click solution to marking important moments from all devices and by allowing everyone to contribute to the editing of files.

From Analog to Digital. Despite the increase of digital material, paper-based documents and pen-and-paper-note-taking are part of current work practices and meetings, too. However, effective sharing can mainly be done with digital material. Therefore, smooth transition between paper-based and digital documentation appears important. In our collaboration environment we try to support this by providing possibilities for digitalizing paper documents within the room. Our facility includes a scanner and a digital camera with an Eye-Fi memory card that both transfer documents automatically to the shared repository.

Logging Document Handling. No meetings happen without supporting documentation, either digital or printed. As these documents are handled during the meeting, they provide both the content as well as the timeline of the meeting. Through the logging of the usage of the handled documents, memory aids about the content and the events can be created as a byproduct of the work. Meeting participants may refer to topics and concepts presented in earlier meetings and, therefore, the availability of these documents and logs about their handling provide additional support for later retrieval of the past issues. Our collaboration environment includes these logging features. There is also an internet browser interface to accessing the logged activity in a timeline and artifact centered presentation.

Human-Recorded Timestamps. Even though no human resources are needed to produce automatic logging of handled documents, the meaning and accuracy of these "timestamps" may be imprecise and require extended effort in later retrieval. The human participants are capable of spotting conceptually and semantically important 
issues and events. The possibility to record human-created timestamps on these moments in the meetings with content snapshots and context data would enable later retrieval of presentations and notes. In our collaboration environment this is supported by providing an easy two-click solution accessible by all devices for marking these important events and automatically recording the context around the moment with snapshots.

Snapshots. In our collaboration environment the "snapshot" concept around important events provides means for recording and retrieval of past issues. The events around timestamps provide both content and context for the timestamp that is the point-of-interest in the meeting timeline. Recording the presentation context (screenshots) as well as the physical context (overall picture of the space) provides means for navigating the meeting timeline afterwards. Additional content-temporal context is stored through audio recording as most of the elaboration on the topics dealt within a meeting happens through discussions.

\section{Discussion}

Our results indicate that there are not well established common practices for capturing, storing, and saving information created in collaborative meetings. Even very basic activities such as note-taking are, after all, not that widely performed. As a consequence of this lack of basic activities, there are also no advanced practices and tools in retrieving data for later use. Due to these, we see many opportunities in developing the interactive collaborative environments further by examining the group memory support functions.

The results of our note-taking and retrieval study are based on a rather small sample. Despite this, however, many of the results appear to be well aligned with other recent studies in the field [5-6]. These relate to the very basic issues such as the lack of meeting minutes as well as their accuracy and personal nature.

In addition to these findings on memory support functionality, there are also interesting extensions worth examining in further studies. Until recently, extensive recording of audio has not widely been technically feasible. Contemporary solutions, however, enable even the recordings of whole meetings. These make it possible to rewind to an earlier discussion and statements and make shorter snapshot recordings (e.g.[8]).

While the technological developments seem to offer straightforward solutions for memory functions, the human practices constrain the possibilities. Therefore, we aim at continuing our studies from the user-centered viewpoint in the development of memory support functionality of interactive collaboration environments. Our future work deals with experiences and evaluation of this functionality.

Acknowledgements. The work reported in this paper has been funded within the Spaces and Places programme by the Finnish Funding Agency for Technology and Innovation (Tekes) and the collaborating organizations. The authors would like to thank Mikael Runonen and Mika P. Nieminen for contributing to the data gathering and Nick Eriksson for technical implementation. 


\section{References}

1. Haller, M., Leitner, J., Seifried, T., Wallace, J.R., Scott, S.D., Richter, C., Brandl, P., Gokcezade, A., Hunter, S.: The NiCE Discussion Room: Integrating Paper and Digital Media to Support Co-Located Group Meetings. In: CHI 2010, Proceedings of the SIGCHI Conference on Human Factors in Computing Systems, pp. 609-618. ACM, Atlanta (2010)

2. Wigdor, D., Jiang, H., Forlines, C., Borkin, M., Shen, C.: WeSpace: the design development and deployment of a walk-up and share multi-surface visual collaboration system. In: CHI 2009, Proceedings of the SIGCHI Conference on Human Factors in Computing Systems, pp. 1237-1246. ACM, New York (2009)

3. Johanson, B., Fox, A., Winograd, T.: The Interactive Workspaces Project: Experiences with Ubiquitous Computing Rooms. IEEE Pervasive Computing 1(2), 67-74 (2002)

4. Geyer, W., Richter, H., Fuchs, L., Frauenhofer, T., Daijavad, S., Poltrock, S.: A team collaboration space supporting capture and access of virtual meetings. In: 2001 International ACM SIGGROUP Conference on Supporting Group Work, pp. 188-196. ACM, New York (2001)

5. Jaimes, A., Omura, K., Nagamine, T., Hirata, K.: Memory cues for meeting video retrieval. In: 1st ACM Workshop on Continuous Archival and Retrieval of Personal Experiences, pp. 74-85. ACM, New York (2004)

6. Whittaker, S., Tucker, S., Swampillai, K., Laban, R.: Design and evaluation of systems to support interaction capture and retrieval. Personal and Ubiquitous Computing 12, 197-221 (2008)

7. Biehl, J.T., Baker, W.T., Bailey, B.P., Tan, D.S., Inkpen, K.M., Czerwinski, M.: IMPROMPTU: A New Interaction Framework for Supporting Collaboration in Multiple Display Environments and Its Field Evaluation for Co-located Software Development. In: CHI 2008 Proceedings of the SIGCHI Conference on Human Factors in Computing Systems, pp. 939-948. ACM, New York (2008)

8. Hunter, S., Maes, P., Scott, S., Kaufman, H.: MemTable: An Integrated System for Capture and Recall of Shared Histories in Group Workspaces. In: CHI 2011 Proceedings of the SIGCHI Conference on Human Factors in Computing Systems, pp. 3305-3314. ACM, New York (2011)

9. Plaue, C., Stasko, J., Baloga, M.: The Conference Room as a Toolbox: Technological and Social Routines in Corporate Meeting Spaces. In: 4th International Conference on Communities and Technologies, C\&T 2009, pp. 95-104. ACM, New York (2009) 